\title{
EL FENÓMENO DEL ACOSO LABORAL ENTRE LOS TRABAJADORES DE LA UNIVERSIDAD
}

\author{
Fernando Justicia Justicia \\ Juan L. Benítez Muñoz \\ Eduardo Fernández de Haro \\ Ana G. Berbén ${ }^{\mathfrak{}}$
}

\begin{abstract}
RESUMEN. El objetivo del artículo es realizar una descripción del fenómemo mobbing -acoso laboral- en el contexto de la universidad. Los trabajos realizados sobre este problema no son muy prolíficos lo que puede explicar la gran dificultad para caracterizar el fenómeno en el ámbito universitario. En el presente trabajo presentamos los niveles de incidencia del acoso laboral entre la población universitaria, los tipos de acoso más frecuentes y los efectos producidos en las víctimas, con la finalidad de caracterizar y definir el problema. Al mismo tiempo, intentamos conocer la influencia de determinadas variables como la edad, género y tiempo de victimización. En este sentido, y de acuerdo con otros estudios realizados, encontramos diferencias estadísticas en función del género de las víctimas. Del mismo modo, los resultados apuntan que la frecuencia y duración del acoso laboral afectan negativamente a las víctimas provocando un empeoramiento de los efectos derivados del acoso.
\end{abstract}

Palabras-clave: acoso laboral, universidad, efectos.

\section{O FENÔMENO MOBBING ENTRE OS TRABALHADORES DA UNIVERSIDADE}

RESUMO. O objetivo deste artigo é realizar uma descrição do fenômeno mobbing -tormento e persecução no lugar do trabalho- no contexto da universidade. Há poucos trabalhos realizados sob este problema nos contextos de educação superior o que pode explicar a grande dificuldade para caracterizar o fenômeno no âmbito universitário. Neste artigo apresentamos os níveis de incidência entre a população universitária, os tipos de mobbing mais freqüentes e os efeitos produzidos nas vítimas com a finalidade de caracterizar e definir o problema. Ao mesmo tempo, tentamos conhecer a influência de determinadas variáveis tais como a idade, gênero ou o tempo de exposição ao fenômeno. Neste sentido, e em concordância com outros estudos realizados, encontramos diferenças estatísticas em função do gênero das vítimas. De igual forma, os dados apontam que a freqüência e duração da situação de persecução no trabalho afetam negativamente as vítimas provocando um agravamento dos efeitos derivados do mobbing.

Palavras-chave: mobbing, universidade, efeitos.

\section{THE PHENOMENON OF MOBBING AMONG UNIVERSITY EMPLOYEES}

\begin{abstract}
* Catedrático del Dpto. de Psicologia Evolutiva y de la Educación. Universidad de Granada.
\# Profesor Colaborador del Dpto. de Psicología Evolutiva y de la Educación. Universidad de Granada.

II Profesor Titular del Dpto. de Psicología Evolutiva y de la Educación. Universidad de Granada.

x Profesora Ayudante del Dpto. de Psicología Evolutiva y de la Educación. Universidad de Granada.
\end{abstract}

ABSTRACT. The objective of this article is to carry out a description of mobbing -workplace bullying- within the university context. There are few studies which have focused their interest in this problem into the higher educational sector and it can explain the great difficulties for characterizing and defining the phenomenon into the university context. In this work, we present results about incidence taxes, most frequent mobbing behaviours and the effects on victims in order to characterize and define the problem. At the same time, we try to know about the influence of specific variables as age, gender or time of exposition to mobbing. In this sense, and in agreement with other studies, we found statistical differences related to victims' 
gender. In the same way, the results point out that frequency and duration of mobbing situations affects negatively to victim provoking an aggravation of the effects derived from mobbing.

Key words: Mobbing, university, effects.

La violencia en el lugar de trabajo ha recibido poca atención por parte de los investigadores (Jennifer, Cowie \& Ananiadou, 2003; Rayner, 1997). Sin embargo, el interés sobre el tema ha aumentado en los últimos años dado que, después del entorno familiar, el contexto laboral y las relaciones interpersonales que en él se establecen son las más importantes para el desarrollo del individuo (Björkqvist, Österman \& Hjelt-Bäck, 1994). Varios estudios ponen de manifiesto que unas malas relaciones con los compañeros de trabajo afectan negativamente al bienestar y salud del trabajador (Mikelsen \& Einarsen, 2002; Zapf \& Gross, 2001). Höel, Sparks y Cooper (2001) señalan la existencia de factores que provocan un ambiente hostil en el lugar de trabajo, entre los que se hallan los conflictos interpersonales $y$ las malas relaciones entre compañeros y que identifican como detonantes del acoso (mobbing) y del maltrato (bullying) en el ámbito laboral. Un primer problema surge cuando abordamos la problemática de la violencia en el lugar de trabajo, y es que no hay consenso ni en cuánto al término ni a la definición del problema (Lewis \& Orford, 2005; Quine, 1999; Zapf \& Einarsen, 2001).

El término y la definición de bullying hacen referencia a acciones intencionales dirigidas a hacer daño, que pueden ser de carácter físico, verbal y/o psicológico, que son frecuentes y duraderas, y en las que se hace necesario un desequilibrio de poder entre el agresor y la víctima (Neuman, 2000; Olweus, 1993). Por otro lado, el término mobbing, aún haciendo referencia a acciones intencionalmente dañinas, que son frecuentes y duraderas (Einarsen, 2000; Leymann, 1996), no incluye las agresiones físicas entre compañeros, que rara vez tienen lugar entre compañeros de trabajo (Zapf, 1999), ni establece como necesario el desequilibrio de poder entre víctima y acosador. En este sentido, Neuman (2000) indica que el bullying siempre lo realiza alguien con poder (real o ficticio) contra otro que no lo tiene, en tanto que el mobbing, puede ser de superior a inferior, entre iguales, e incluso, de inferiores a superiores (Leymann, 1996). A la vista de lo anterior nos decantamos por la utilización del término acoso moral para hablar del fenómeno dado que engloba ambos términos (mobbing y bullying) tanto etimológicamente como a nivel de contenidos. Hacemos entonces referencia a comportamientos intencionales, hostiles y poco éticos, dirigidos a infligir daños en las víctimas y que son sistemáticos, frecuentes y duraderos (Doyle, 2001; Hirigoyen, 2001; Leymann, 1996; Soares, 2002). Los comportamientos de acoso pueden clasificarse en cinco categorías (Leymann, 1996; Rayner \& Höel, 1997): amenazas al estatus profesional, saturación profesional, desestabilización profesional, abusos personales, y aislamiento social.

Diversos estudios han puesto de manifiesto la incidencia del fenómeno (Höel \& Cooper, 2000a; HSA, 2001; Lewis, 1999; Leymann, 1992; Rayner, 1997) que se mueve entre un $3,5 \%$ y un $25 \%$ en función del país, la definición del problema, la muestra participante, el instrumento de medida, los criterios de evaluación, etc. En el caso específico del sector educativo, los estudios lo señalan como uno de los más afectados. La encuesta europea sobre acoso apunta que el $12 \%$ de los trabajadores acosados pertenecen al sector educativo (Di Martino, Hoel \& Cooper, 2003); el informe irlandés (HSA, 2001) señala una incidencia del $12,1 \%$, colocando a dicho sector como el segundo más afectado, dato apoyado por la investigación de Hubert y Van Veldhoven (2001). Por su parte, Höel y Cooper (2000b) establecen una tasa de incidencia del fenómeno en este sector que asciende a un $15,6 \%$ de los casos. En cuanto a la incidencia del fenómeno en educación universitaria los estudios no son muy prolíficos. Así, Björkqvist, Österman y Hjelt-Bäck (1994) realizaron un estudio sobre acoso laboral entre miembros de la comunidad universitaria situando la tasa de víctimas en un $17 \%$.

Un aspecto de vital importancia en el fenómeno del mobbing se relaciona con los efectos que provoca no sólo al trabajador sino también a la institución en la que trabaja, e incluso a la propia sociedad (Fornés, 2003; Höel \& Cooper, 2000; Höel, Sparks \& Cooper, 2001; ILO, 2003; Leymann, 1996; Salin, 2003; Soares, 2002). En relación a los daños al trabajador que son los que nos ocupan y los que analizaremos posteriormente, Björkqvist, Österman y Hjelt-Bäck (1994) encontraron que las víctimas de acoso presentan altos niveles de ansiedad, depresión y agresividad. Además, las víctimas señalan el insomnio, la apatía y la falta de concentración como otros problemas derivados de la situación laboral de acoso. En la misma dirección, Einarsen (2000), Vartia (2001) y Höel, Sparks y Cooper (2001), destacan en el 
ámbito individual los efectos sobre la salud mental y física: depresión, ansiedad, estrés, inseguridad, miedo, desmotivación, falta de concentración, desconfianza, insomnio, problemas cardíacos. Del mismo modo, Di Martino, Höel y Cooper (2003) resumen en su estudio que el trabajador ve afectada su salud física, su salud emocional, su actividad profesional y su actividad social.

\section{MÉTODO}

\section{Participantes}

En el estudio participan 325 trabajadores, pertenecientes al personal docente (75\%) y de administración y servicios (25\%) de la Universidad de Granada (Granada, España). La muestra $(62,7 \%$ de hombres y $37,3 \%$ de mujeres) está formada por todos aquellos que respondieron al cuestionario accesible en Internet. La población universitaria a la que se solicitó participar en el estudio fue de 2876 trabajadores, que representaban el total de trabajadores universitarios con disponibilidad de correo electrónico en ese momento. Accedieron y respondieron al cuestionario 325 personas de las 2876 que recibieron notificación del estudio vía correo electrónico, lo que representa un $11,3 \%$ del total de la población universitaria a la que se pidió colaboración.

\section{Instrumento}

Para analizar la incidencia del acoso laboral, así como para caracterizar el fenómeno, fue utilizado el Cuestionario para el Estudio del Acoso Laboral en Contextos Universitarios (Justicia \& cols., 2002). Para su construcción se revisaron otros instrumentos: Leymann Inventory of Psychological Terrorization de H. Leymann (G. de Rivera, 2002); Psychological Workplace Inventory (Björkqvist \& Österman, 1998); Work Atmosphere Scale (Björkqvist \& Österman, 1998); Work Stress Sympton Scale (Björkqvist \& Österman, 1998); Barómetro Cisneros II (Piñuel, 2002); Workplace Relationships Questionnaire (Jennifer, 2001) y Experiencies in Workplace Questionnaire (HSA, 2001). El cuestionario (Cronbach's Alpha $=0,9250$ ) consta de una primera parte con nueve ítems que recaba información sobre género, edad, titulación académica, puesto profesional, experiencia laboral del encuestado, etc.; y una segunda parte que consta de 32 ítems. La segunda parte del cuestionario se divide en cuatro bloques: (a) caracterización de fenómeno (12 ítems); (b) caracterización del acoso según las víctimas (19 ítems); (c) caracterización del acoso según los testigos
(2 ítems); y (d) caracterización del acosador (3 ítems). La validez del instrumento fue testada a través de juicio de seis expertos pertenecientes al Departamento de Psicología Evolutiva y de la Educación y dos expertos en Métodos y Diseños de Investigación de la Universidad de Granada.

\section{Diseño y procedimiento}

Dado que la Universidad de Granada como institución era el objeto de estudio, el proyecto de investigación se presentó al Equipo de Gobierno de la universidad para su conocimiento y apoyo, solicitando la correspondiente autorización para llevarlo a cabo. Tomamos la decisión de presentar el cuestionario a través de Internet. Para tal fin, se construyó una página Web (www.estudiosmobbing.com), elaborada por una empresa especializada, en la cual se daba información sobre la finalidad del estudio y el modo de acceso al cuestionario.

La página de presentación contenía información sobre los coordinadores del proyecto, los objetivos perseguidos y hacía una breve descripción del fenómeno del acoso laboral. La última parte hacía referencia al carácter confidencial de los datos y al procedimiento a seguir por todos los interesados en responder al cuestionario. El acceso al cuestionario estaba restringido por un código de acceso que fue remitido al personal de la universidad a través de correo electrónico. El control de acceso estaba doblemente motivado. Primero, para preservar la identidad $y$ el anonimato de cuantos pudieran participar con sus respuestas en el estudio. Segundo, para garantizar que sólo respondían trabajadores de la universidad y que lo hacían una sola vez. Una vez rellenado y enviado el cuestionario se invalidaba el código y las respuestas de los participantes pasaban a la base de datos.

La presentación del cuestionario en pantalla se hizo ítem a ítem sin número de orden que los identificara. No era posible pasar de un ítem al siguiente sin haber cumplimentado correctamente los datos mínimos requeridos. El número de ítems contestados por participante dependía de la naturaleza de las preguntas contestadas.

\section{RESULTADOS}

\section{Percepción de victimización entre los participantes}

De los 325 participantes, el 24,08\% se auto percibe como víctima de acoso laboral alguna vez. Sin embargo, es necesario atender a criterios estadísticos de frecuencia (1 vez por semana) y duración 
(experiencia de acoso de al menos 6 meses) del acoso para poder ofrecer tasas de acoso reales. Así, el porcentaje de víctimas se reduce hasta el $11 \%$ de la población encuestada. En cuanto al género de las víctimas, los datos indican un mayor número de víctimas entre los hombres $(52,8 \%)$ que entre las mujeres (47,2\%). Sin embargo, tenemos que considerar que las mujeres representan el $37,3 \%$ y los hombres el $62,7 \%$ del total de la muestra. Para ello, y realizando el análisis a través de la comparación de medias (ANOVA) por razón de género, permite comprobar que las mujeres son más acosadas que los hombres y que la diferencia resulta estadísticamente significativa $[F(1)=3,986, p<0,05)]$. En cuanto a la edad, el acoso laboral se concentra básicamente en personas entre los 36 y 55 años en un 76,4\% de los casos, resultando esta distribución estadísticamente significativa $\left[\kappa^{2}(4)=56.750, \mathrm{p}<0.001\right]$. El dato anterior indica que el acoso laboral afecta a trabajadores con una experiencia laboral media, ya que el tiempo de experiencia de trabajo de las víctimas se sitúa entre los 6 y 20 años de antigüedad en la universidad $(68 \%)$.

\section{Comportamientos de acoso y dirección del acoso}

Las formas más frecuentes de acoso laboral en la universidad son las siguientes: ocultar información $(55,3 \%)$, infravalorar el trabajo $(55,3 \%)$, no tener en cuenta la opinión de las personas $(53,7 \%)$, criticar sin fundamento (46\%), infravalorar el esfuerzo (45,3\%), exclusión social $(41,7 \%)$, limitar las tareas sin justificación (37\%), propagar rumores falsos $(34,7 \%)$, la sobrecarga de trabajo $(31,7 \%)$.

En cuanto a la dirección del acoso, la mayor parte de los encuestados señala como más frecuente la situación de acoso descendente: la que se produce de un superior a un inferior (76,3\%). En la medida en que, las direcciones de acoso no son excluyentes entre sí y un mismo acosador puede llevar a cabo diferentes comportamientos de acoso independientemente de la categoría de la víctima, llama la atención el alto porcentaje de acoso que se produce entre compañeros de mismo estatus o nivel profesional (55,3\%). Finalmente, el acoso ascendente (de inferior a superior) tiene menor incidencia con una tasa del $26,3 \%$.

\section{Efectos del acoso laboral en las víctimas}

Los efectos del acoso son analizados a través de un ítem en el que se presentan diferentes síntomas (32) que el sujeto señala sólo en el caso de sufrirlas (valor 1). Los efectos se clasifican en cuatro áreas: a) salud emocional: disminución auto confianza, disminución de la autoestima, problemas de motivación, ansiedad, comportamiento agresivo, depresión, pensamientos de abandono del trabajo, ideas suicidas; b) salud física: insomnio, cansancio, dolores de cabeza, taquicardias, trastornos digestivos, nauseas/vómitos, dolores musculares, temblores/sudores; c) relaciones sociales: problemas con amigos, compañeros, familiares, pareja, alumnos, destinatarios del trabajo, superiores, asilamiento social; y, d) actividad profesional: disminución de la concentración, disminución del rendimiento, disminución de la estancia en el centro, desmotivación, falta de interés por cooperar, petición de traslado, petición de baja laboral, disminución del horario laboral.

En primer lugar, y para estudiar la relación entre los efectos y la edad de las víctimas se analizan los resultados utilizando la prueba de Correlación de Spearman con significación de tipo bilateral. No se encuentran diferencias en ninguna de las áreas analizadas, a excepción de la profesional en la que encontramos algunas diferencias. Las correlaciones más importantes encontradas en esta área muestran que conforme aumenta la edad de las víctimas es menos frecuente que disminuya su rendimiento $(r=-$ $0,203)$ y que pidan bajas laborales $(r=-0,305, p<0,01)$ como consecuencia del acoso sufrido.

En relación a los efectos del mobbing y el género de las víctimas (Figura 1) se observaron diferencias estadísticas significativas. Para estudiar las diferencias entre hombres y mujeres hemos utilizado la prueba no paramétrica U de Mann Whitney. Obteniendo diferencias significativas en las áreas emocional, física y profesional.

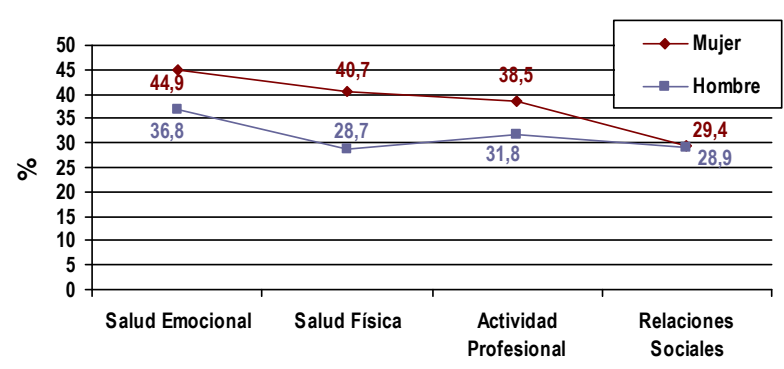

Figura 1. Efectos del Acoso y Género de las Víctimas.

En el área emocional, las mujeres presentan mayores niveles de comportamiento agresivo (MannWhitney $\mathrm{U}$ test; $p<0,05)$ y más altas tasas de estados depresivos (Mann-Whitney $\mathrm{U}$ test; $p<0,05$ ) que los hombres. En el área física el cansancio generalizado de las mujeres es significativamente superior al que muestran los hombres (Mann-Whitney U test; $p<0,05$ ), al igual que son significativamente más comunes entre las mujeres los dolores de cabeza (Mann-Whitney U 
test; $p<0,001)$. En cuanto a los efectos en el área profesional, se encuentran diferencias significativas en la utilización de las bajas laborales siendo más numerosas entre las mujeres (Mann-Whitney U test; $p<0,001)$.

Para finalizar con la comparación entre mujeres y hombres, consideramos importante mencionar otras diferencias encontradas que aunque no llegan a ser significativas, marcan tendencias. Las diferencias se encuentran en la disminución del rendimiento laboral, mayor en las mujeres que en los hombres (MannWhitney $\mathrm{U}$ test; $p=0,07)$ y en el padecimiento de trastornos digestivos también más comunes en las mujeres (Mann-Whitney U test; $p=0,068$ ).

\section{Efectos, frecuencia y duración del acoso}

Las víctimas son clasificadas según criterios de frecuencia y duración en: victima pura si se cumple con ambos criterios, y víctima ocasional si se incumple con alguno de ellos (Jennifer, Cowie \& Ananiadou, 2003). La distribución general de los efectos en cada una de las áreas analizadas emocional, física, profesional y relaciones socialesmuestra un mayor impacto en las victimas puras que en las ocasionales en todas las categorías (Figura 2).

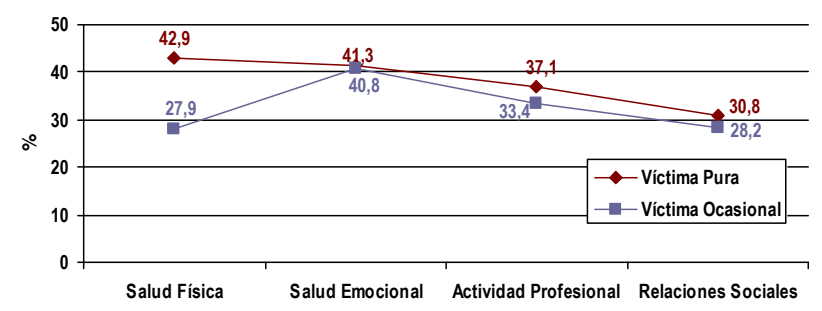

Figura 2. Efectos y Tipo de Víctimas.

En relación con la salud física, existen diferencias significativas con respecto a cansancio generalizado y los trastornos digestivos que son más comunes en las victimas puras (Mann-Whitney $\mathrm{U}$ test; $p<0,05$ ). En el área emocional observamos que los estados de ansiedad y los pensamientos de abandono del trabajo son sufridos significativamente más por las victimas puras (Mann-Whitney U test; $p<0,001$ ). Por otro lado, las víctimas ocasionales padecen más depresiones y más ideas suicidas (Mann-Whitney $\mathrm{U}$ test; $p<0,05$ ). Otro efecto que sufren de forma diferente unos $y$ otros, aunque no es estadísticamente significativo, son los comportamientos agresivos, que son más usuales entre las víctimas ocasionales (Mann-Whitney U test; $p=0,056)$. Las diferencias significativas encontradas en relación con la actividad profesional ponen de manifiesto que las victimas puras muestran mayor desmotivación hacia el trabajo (Mann-Whitney U test; $p<0,05)$ y un mayor desinterés por cooperar con los compañeros (Mann-Whitney $\mathrm{U}$ test; $p<0,05$ ). Finalmente, los resultados muestran que en el área de las relaciones sociales las victimas puras presentan mayor aislamiento social (Mann-Whitney U test; $p<0,05)$; mayor deterioro de las relaciones personales con la pareja (Mann-Whitney U test; $p<0,05$ ), con los compañeros de trabajo (Mann-Whitney $U$ test; $p<0,001$ ), y con personas de cargos superiores (MannWhitney $\mathrm{U}$ test; $p<0,05)$. Sin embargo, ven menos afectadas sus relaciones con los destinatarios de su trabajo (Mann-Whitney U test; $p<0,001$ ).

\section{DISCUSIÓN Y CONCLUSIONES}

Los datos obtenidos revelan que un $11 \%$ de los participantes en el estudio dicen sufrir de forma frecuente y duradera acoso en su lugar de trabajo. Nuestros hallazgos no discrepan de los obtenidos por otros estudios e incluso la tasa de incidencia es menor a la encontrada por Di Martino, Hoel y Cooper (2003) y la HSA (2001) quienes la sitúan en torno al $12 \%$. Sin embargo, ninguno de ellos proporciona datos sobre la universidad. En este sentido, sólo Björkqvist, Österman y Hjelt-Bäck (1994), en su estudio sobre acoso laboral entre trabajadores universitarios proporcionan un porcentaje superior al que ofrecemos nosotros y que sitúan en un $17 \%$.

En cuanto a la relación acoso laboral y género, hay estudios que no establecen diferencias de género apuntando que el fenómeno afecta por igual a hombres y mujeres (Einarsen \& Skogstad, 1996; Rayner, 1997), en tanto que otros establecen diferencias entre ambos colectivos (Lewis \& Ordford, 2005; Ólaffson \& Jóhannsdíttir, 2004; Rayner, 1997). Según nuestros datos el porcentaje de mujeres que sufre acoso laboral es significativamente superior al de hombres. Este dato es más consistente, si cabe, cuando observamos que en el estudio las mujeres, independientemente del colectivo profesional al que pertenecen, sufren acoso con más frecuencia que los hombres. En el trabajo de Björkqvist y colaboradores (1994) con empleados universitarios también se confirma que entre las víctimas existe una mayor tasa de mujeres $(24,4 \%)$ que de hombres $(16,9 \%)$.

En relación a los efectos provocados por el mobbing Di Martino, Höel y Cooper (2003) y Martín, Pérez y López (2002) establecen que las áreas en que las víctimas se ven afectadas son esencialmente: la salud física, la salud emocional, el desempeño profesional y el ámbito social de la víctima. Los resultados obtenidos de la muestra participante en 
nuestro estudio revelan que son éstas las áreas afectadas, al mismo tiempo que informan de otros aspectos no recogidos en el cuestionario y que son susceptibles de ser clasificados como efectos del acoso laboral. En relación con las áreas emocional y física, los problemas de motivación, autoestima, insomnio y cansancio generalizado, son los más frecuentes entre las víctimas como también apuntaron Einarsen (2000), Höel, Sparks y Cooper (2001), y Mikkelsen y Einarsen (2002). En lo relativo al desempeño profesional y coincidiendo con los datos ofrecidos por Fornés (2003) y Leymann (1996), la disminución del rendimiento y la desmotivación hacia el trabajo son los efectos que con mayor frecuencia indican los participantes en el estudio. Finalmente, los problemas de relación con la pareja y los compañeros son los más importantes para las víctimas.

De nuestros datos destacamos cuatro aspectos. Primero, hay diferencias significativas entre hombres $\mathrm{y}$ mujeres en cuanto a daños en la salud física y emocional, siendo más importantes en las mujeres que en los hombres. Segundo, ambos colectivos presentan patrones diferentes en la importancia que confieren a las consecuencias del acoso en los diferentes ámbitos; mientras que las mujeres ven más afectada la salud física, la salud emocional, el desempeño profesional y las relaciones sociales, los hombres invierten este orden. Los datos difieren de los encontrados por Björkqvist y cols. (1994) quienes no observaron incidencia del género en los efectos del acoso entre los empleados universitarios. Tercero, la gravedad de las consecuencias del acoso varía en función del tiempo y duración del período de victimización (Björkqvist \& cols., 1994; Soares, 2002; Vartia, 2001). Así, para Björkqvist y cols. las victimas puras tienen tasas más altas de depresión, ansiedad y agresividad que las ocasionales. En nuestro caso, dependiendo del tiempo de victimización, las diferencias son significativas en varios aspectos ya que las victimas puras: (a) muestran más comportamientos agresivos; (b) ven como se empobrecen las relaciones sociales; y (c) se aíslan más de sus compañeros, sus familiares y su pareja. Este hallazgo es similar al encontrado por Mikkelsen y Einarsen (2002) quienes afirman que la exposición al mobbing incrementa los problemas físicos, psicológicos y psicosomáticos. Cuarto, la influencia de la variable edad sobre los efectos provocados por el mobbing no es significativa.

A pesar de todos los datos quedan preguntas sin contestar y los resultados obtenidos han de ser tomados con cautela. En primer lugar, hemos de señalar que la muestra de participantes pertenece a una universidad y sería necesario el estudio de mayor número de universidades para afianzar las tendencias de resultados observados en este estudio. En segundo lugar, y asociado a la limitación anterior, algunos de los aspectos analizados sólo consideran las respuestas de las víctimas, lo que aún reduce más si cabe la generalización de los resultados obtenidos. Aunque esta segunda limitación siempre estará presente cuando se quiera hacer un estudio específico sobre víctimas. Además, sería interesante realizar estudios que evalúen el mantenimiento de los efectos provocados por el fenómeno; es decir, investigaciones que realicen un seguimiento de los efectos a largo plazo del acoso laboral. Al mismo tiempo se podrían analizar los efectos a largo plazo en hombres y mujeres. Del mismo modo, sería interesante realizar un estudio como el de Quine (1999) en el que se analizan no sólo las consecuencias del acoso laboral para las víctimas de acoso, sino también para los testigos de estas situaciones, ya que como afirma este autor también resultan afectados cuando presencian acoso laboral, aspecto que incide negativamente en su desarrollo profesional.

El análisis de los efectos del acoso laboral no ha de perder de perspectiva la influencia que tales efectos tiene sobre el desempeño profesional del profesorado y del personal de administración y servicios, ya que claramente afectarán a la calidad de la Educación Superior. Y es que si abogamos por una mejora de la calidad de la Educación Superior está debe pasar, inexorablemente, por la creación de contextos de trabajo saludables en los que la realización profesional esté garantizada. Así, la reducción de riesgos psicosociales y el establecimiento de condiciones laborales adecuadas entre los profesionales de la universidad se presentan como condiciones necesarias para lograr dicha calidad. Se precisa por tanto, del establecimiento de medidas de intervención desde las instituciones universitarias que señalen claramente qué hacer y cómo combatir el fenómeno del acoso laboral: protocolos de diagnóstico y evaluación del fenómeno, campañas de sensibilización e información sobre el problema, formación en resolución de conflictos, formación específica para el desarrollo del trabajo cooperativo, asesoramiento jurídico por parte de la institución, etc. La adopción de tales protocolos de actuación ayudará a diagnosticar, evaluar y solucionar situaciones de acoso laboral antes de que provoquen daños en aquellos que sufren el problema.

\section{REFERÊNCIAS}

Björkqvist, D. \& Österman, K. (1998). Scales for Research on Interpersonal Relations. Pro Facultate, 4. 
Björkqvist, D., Österman, K. \& Hjelt-Bäck, M. (1994). Aggression among university employees. Aggressive Behavior, 20, 173184.

Di Martino, V. P., Höel, H. \& Cooper, C. (2003). Preventing violence and harassment in the workplace. Dublin: European Foundation for the improvement of living and working conditions.

Doyle, E. (2001). Dignity at work: The challenge of workplace bullying. Dublin: Stationary Office.

Einarsen, S. (2000). Harassment and bullying at work: A review of the Scandinavian approach. Aggression and Violent Behavior, 5(4), 379-401.

Einarsen, S. \& Skogstad, A. (1996). Bullying at work: Epidemiological findings in public and private organizations. European Journal of Work and Organizational Psychology, 5, 185-201.

Fornés, J. (2003). Acoso psicológico en el mundo laboral: Responsabilidades y control. IV Congreso Virtual de Psiquiatría, Interpsiquis.

González de Rivera, J. L. (2002). El maltrato psicológico. Madrid: Espasa Calpe.

Hirigoyen, M. F. (2001). El acoso moral en el trabajo. Barcelona: Paidós Contextos.

Höel, H. \& Cooper, C. (2000a). Workplace bullying in Britain. Employee Health Bulletin, 14, 9-14.

Höel, H. \& Cooper, C. (2000b). Destructive conflict and bullying at work. Manchester: British Occupational Health Research Foundation.

Höel, H., Sparks, K. \& Cooper, C. (2001). The cost of violence/stress at work and the benefits of a violence/stress-free working environment. Geneva: International Labour Organization.

HSA - Health and Safety Authority (2001). Dignity at work: The challenge of workplace bullying. Dublin: Health and Safety Authority.

Hubert, A. \& Van Veldhoven, M. (2001). Risk sectors for undesirable behaviour and mobbing. European Journal of Work and Organizational Psychology, 10(4), 415-424.

ILO - International Labour Organization. (2003). Introduction to violence at work: InFocus Programme on Safety and Health at Work and the Environment. Retrieved June 11, 2005, from http://www.ilo.org/public/english/protection/safework/violence/ intro.htm

Jennifer, D. (2001). Workplace Relationships Questionnaire. University of Surrey. Unpublished paper.

Jennifer, D., Cowie, H. \& Ananiadou, K. (2003). Perceptions and experience of Workplace Bullying in Five Different Working Populations. Aggressive Behaviour, 29, 489-496.

Justicia, F., Fernández, E., Benítez, J. L., Villena, M. D., García, A. \& Caurcel, M. J. (2002). Cuestionario sobre Acoso Laboral en Contextos Universitarios. Unpublished Scientific Paper. Registro n ${ }^{\circ}$ GR-1196-02.
Lewis, S. \& Orford, J. (2005). Women's experiences of workplace bullying: Changes in social relationships. Journal of Community \& Applied Social Psychology, 15, 29-47.

Lewis, D. (1999). Workplace Bullying: Interim findings of a study in further and higher education in Wales. International Journal of Manpower, 20(1), 106-118.

Leymann, H. (1992). Från mobbning till utslagning $i$ arbetslivet. Stockholm: Publica.

Leymann, H. (1996). The content and development of mobbing at work. European Journal of Work and Organizational Psychology, 5(2), 165-184.

Martín, F., Pérez, J. \& López, J. (2002). El hostigamiento psicológico en el trabajo: Mobbing. Recuperado el 10 de mayo de 2004, de http://www.mobbing.nu/476.htm.

Mikelsen, E. \& Einarsen, S. (2002). Relationships between exposure to bullying at work and psychological and psychosomatic healt complaints: The role of state negative affectivity and generalized self-efficacy. Scandinavian Journal of Psychology, 43, 397-405.

Neuman, J. (2000). Injustice, stress and bullying can be expensive!. In Workplace Bullying Conference, Oakland, California. Retrieved December 04, 2002, from http://www. bullybusters.org.

Ólaffson, R. \& Jóhannsdíttir, H. (2004). Coping with bullying in the workplace: The effect of gender, age and type of bullying. British Journal of Guidance and Counsellying, 32(3), 319-333.

Olweus, D. (1993). Bullying at school: What we know and what we can do. Cambridge: Blackwell.

Piñuel, I. (2002). La incidencia del mobbing o acoso psicológico en el trabajo en España. Informe Cisneros II sobre violencia en el entorno laboral. Universidad de Alcalá.

Quine, L. (1999). Workplace bullying in NHS community trust: Staff questionnaire survey. British Medical Journal, 318(7178), 228-332.

Rayner, C. (1997). The incidence of workplace bullying. Journal of Community and Applied Social Psychology, 7, 199-208.

Rayner, C. \& Höel, H. (1997). A summary review of literature relating to workplace bullying. Journal of Community and Applied Social Psychology, 7, 181-191.

Salin, D. (2003). Ways of explaining workplace bullying: A review of enabling, motivating and precipitatin structures and processes in the work environment. Human Relations, 56(10), 12131232.

Soares, A. (2002). Bullying: When work becomes incident. Recuperado el 06 de Septiembre de 2003, de http://www.er.uqam.ca/nobel/r13566/document/bullying\%20re port\%20soares.pdf.

Recebido em 03/07/2006 Aceito em 28/06/2007

Endereço para correspondência: Juan Luís Benítez Muñoz. Dpto. Psicología Evolutiva y de la Educación de la Universidad de Granada. Fac. Ciencias de la Educación. Campus Cartuja s/n, 18071, Granada, España.E-mail: jlbenit@ugr.es 